\author{
Nelleke van Sluisveld \\ Gijs Hesselink \\ Johannes Gerardus \\ van der Hoeven \\ Gert Westert \\ Hub Wollersheim \\ Marieke Zegers
}

\section{Improving clinical handover between intensive care unit and general ward professionals at intensive care unit discharge}

Received: 22 October 2014

Accepted: 14 January 2015

Published online: 12 February 2015

(C) The Author(s) 2015. This article is published with open access at Springerlink.com

Take-home message: Liaison nurses and handover forms have a positive effect on the quality of clinical handover between ICU and ward healthcare professionals at patient discharge. Researchers and clinicians considering to conduct an evaluation of an improved handover process should use robust designs to strengthen the evidence on this topic.

Electronic supplementary material The online version of this article (doi:10.1007/s00134-015-3666-8) contains supplementary material, which is available to authorized users.

N. van Sluisveld (®) - G. Hesselink · G. Westert · H. Wollersheim - M. Zegers IQ healthcare, Radboud Institute for Health Sciences, Radboud University Medical Center, 9101, 6500 HB Nijmegen,

The Netherlands

e-mail: nelleke.vansluisveld@ radboudumc.nl

J. G. van der Hoeven

Department of Intensive Care Medicine, Radboud University Medical Center, 9101, 6500 HB Nijmegen, The Netherlands
Abstract Purpose: To systematically review and evaluate the effectiveness of interventions in order to improve the safety and efficiency of patient handover between intensive care unit (ICU) and general ward healthcare professionals at ICU discharge. Methods: PubMed, CINAHL, PsycINFO, EMBASE, Web of Science, and the Cochrane Library were searched for intervention studies with the aim to improve clinical handover between ICU and general ward healthcare professionals that had been published up to and including June 2013. The methods for article inclusion and data analysis were pre-specified and aligned with recommendations outlined in the PRISMA guideline. Two reviewers independently extracted data (study purpose, setting, population, method of sampling, sample size, intervention characteristics, outcome, and implementation activities) and assessed the quality of the included studies.

Results: From the 6,591 citations initially extracted from the six databases, we included 11 studies in this review. Of these, six $(55 \%)$ reported statistically significant effects. Effective interventions included liaison nurses to improve communication and coordination of care and forms to facilitate timely, complete and accurate handover information. Effective interventions resulted in improved continuity of care (e.g., reduced discharge delay) and in reduced adverse events. Inconsistent effects were observed for use of care, namely, reduction of length of stay versus increase of readmissions to higher care. No statistically significant effects were found in the reduction of mortality. The overall methodological quality of the 11 studies reviewed was relatively low, with an average score of 4.5 out of 11 points. Conclusions: This review shows that liaison nurses and handover forms are promising interventions to improve the quality of patient handover between the ICU and general ward. More robust evidence is needed on the effectiveness of interventions aiming to improve ICU handover and supportive implementation strategies.

Keywords Clinical handover . Transitional care - Intensive care . Patient safety - Quality of care . Systematic review

\section{Introduction}

Efficient use of intensive care units (ICUs) has become a top priority of hospitals worldwide as a result of the increased pressure on hospital budgets $[1,2]$. An optimal patient flow is critical to ensure a high quality of care, given that ICUs are often subject to forward pressure from various internal sources, such as emergency departments 
or operating theaters [3, 4]. Early discharge from the ICU to the general ward is one strategy that can be used to relieve this pressure, but the successful implementation of this strategy requires close cooperation between a variety of healthcare professionals across different clinical settings [4-7].

A patient's discharge from one specialty to another is a high-risk event in the care process and one where poor clinical handover between healthcare professionals leads to preventable errors and adverse events $[8,9]$. Patients discharged from the ICU are particularly vulnerable to poor handovers due to the complex physiology of their health condition and the significant decrease in monitoring which occurs upon the transfer of these patients to a general ward [10]. These factors are particularly relevant for patients subjected to early discharge policies $[7$, $11]$.

Despite the availability of professional guidelines for ICU discharge [12-14], the quality of clinical handover practices varies between ICUs [15]. Several studies have identified deficits in the communication, coordination of care and information exchange between ICU and ward healthcare professionals [16-20]. These factors increase the risk of suboptimal ICU discharge and may result in severe adverse events, ICU readmissions, and increased mortality $[6,21,22]$. In a study conducted in the USA in 2003, Nishi and colleagues reported that $37.3 \%$ of the ICU readmissions within $48 \mathrm{~h}$ were potentially preventable $[16,23]$. Based on available data, it is estimated that a reduction of the readmission rate by $1 \%$, incorporating an overall mean ICU stay of 6.6 days, could save the U.S. government $\$ 1.4$ billion per year $[1,23$ 25].

There are several strategies to improve clinical handovers between ambulance crew and emergency department [26, 27], between shifts [28, 29], and between the hospital and community setting [20,30], as well as postoperative handovers [31]. However, a comprehensive evaluation of the effectiveness of interventions with the aim to improve inter-specialty handovers from the ICU to a general hospital ward is lacking. Niven and colleagues recently reviewed the effect of transition programs for patients discharged from an ICU which focused on post-ICU discharge interventions and excluded studies with a neonatal or pediatric population [9]. Better insight into effective interventions could guide healthcare professionals and policy-makers in the development and implementation of polices aimed at reducing patient mortality rates and costly readmissions [32-34].

The purpose of the study reported here was to systematically review interventions with the aim to improve the quality of patient handover between ICU and general ward healthcare professionals at ICU discharge and to evaluate the overall effects of these interventions.

\section{Methods}

The criteria for article inclusion and data analysis were pre-specified [35], and the protocol followed is given in Electronic Supplementary Material (ESM) 1. We followed the recommendations outlined in the Preferred Reporting Items for Systematic Reviews and MetaAnalyses (PRISMA) Statement [36].

\section{Data sources and searches}

Using specific search terms (for details, see ESM Table 1), we searched for full-text intervention studies in the following databases: PubMed (including MEDLINE), CINAHL, PsycINFO, EMBASE, Web of Science, and the Cochrane Library. There were no restrictions based on publication date or language, but the presence of an English abstract was considered to be important. The authors' personal files, references from included studies, and bibliographies of previously published related reviews were also searched to identify additional relevant studies (snowballing) [9, 10, 37].

\section{Study selection}

Two researchers (NS and GH) independently screened the titles and abstracts of all studies identified by the search strategy for their eligibility. A study had to meet all of the following inclusion criteria to be included in the review:

1. Inclusion of patients or healthcare professionals involved in the handover from the ICU to a stepdown unit or ward.

2. Inclusion of an intervention explicitly describing one or more components that aimed to improve the handover of care between healthcare professionals from the ICU and those of a step-down unit or general ward.

3. Study design was experimental or quasi-experimental, such as a (cluster) randomized controlled trial, cohort study, or a non-controlled before-after study.

4. There was at least one process or outcome measure addressing the quality or safety of the discharge.

Studies not available in full-text format were excluded. When the title and abstract did not clearly indicate whether the inclusion criteria were met, a full-text copy was retained and reviewed.

The full text of the potentially relevant studies were retrieved and reviewed by two researchers (NS, GH). The inclusion criteria were applied a second time, and a final set of studies was identified for data extraction. Disagreement on inclusion was resolved by discussion; when 
no consensus could be reached, a third researcher (MZ) made the final decision.

\section{Data extraction}

Data from each study meeting the inclusion criteria were independently extracted by two researchers (NS, GH) using a pre-designed form modified from a checklist developed by Grimshaw and colleagues [38]. The extracted data described the objectives, underlying theorybased concepts, setting, study population, intervention characteristics, implementation activities, process evaluation, and outcome measures. Outcomes were divided into four pre-specified groups by the two researchers separately as: (1) use of care (e.g., ICU readmissions), (2) continuity of care (e.g., information accuracy), (3) adverse events, and (4) mortality. Any disagreement between the two researchers was resolved by discussion.

\section{Quality assessment}

The methodological quality was assessed by two researchers (NS, GH) independently. To ensure standardized scoring, we used a standardized form adapted from the Cochrane Effective Practice and Organization of Care (EPOC) Group's Risk of Bias Criteria [39]. Methodological quality was assessed on 11 criteria, including (1) whether studies used random and concealed allocation, (2) whether the studies documented similar baseline characteristics and outcomes between the intervention and control group, (3) whether the studies described a strategy for handling missing data, (4) the likelihood of contamination between study groups, and (5) whether the criteria were free from selective outcome reporting. The decision on whether the criteria were fulfilled was resolved by discussion, or a final decision was made by the third researcher (MZ). Studies were given 1 point for each fulfilled criterion, with a maximum of 11 points. If information was inadequate or missing, the criterion was labeled 'unknown' and no point was given.

\section{Data synthesis and analysis}

The study outcomes, such as sample size, intervention characteristics, outcome measures, statistical significance, and direction of the effects observed, were assessed by two researchers (NS, GH) and organized in a tabular form.

The interventions were classified by two researchers (NS and GH) based on the definition of continuity of care by Helles $\varnothing$ and colleagues [17]. This classification consists of three elements: (1) the quality of information that is exchanged between healthcare professionals in terms of completeness, accuracy, and clarity; (2) the coordination of care between healthcare professionals in terms of the quality of assessment, planning, and organization of follow-up services and needs; (3) the communication between healthcare professionals in terms of personal and direct contact, accessibility, and timeliness [17, 20].

\section{Results}

Search results

Our initial search identified 6,591 records (Fig. 1), of which 5,268 remained following the exclusion of duplicates. Subsequent screening by title and abstract excluded 5,231 records. The remaining 37 full-text studies were retrieved and reviewed, of which 29 were excluded. Three articles were identified through snowballing. The final set of articles included in the review of consisted of 11 published studies that had undergone full-text extraction [40-50].

\section{Characteristics of included studies}

A summary of the characteristics of the 11 studies included in this review is presented in Table 1 . The study population included neonatal, pediatric, and adult patients and their healthcare professionals. Patients were treated in neonatal, pediatric, general, medical, and/or surgical ICUs situated in various types of hospitals (tertiary, regional, metropolitan, teaching, university/academic, communityteaching, tertiary-referral). Ten studies were single-center studies, and one was conducted across multiple $(n=3)$ hospitals [47]. The sample size per study ranged from 46 to 4,951 participants for the intervention group and from 53 to 1,872 participants for the control group.

The studies reported various outcomes (Table 2), although most studies reported an outcome related to use of care [40, 42-45, 47, 49, 50], mortality [40, 44, 45, 47, $49,50]$, and continuity of care [41, 48-50]. One study reported adverse events as an outcome measure [46]. Statistical significant improvements were observed in two categories: continuity of care (reduced discharge delay, increased perceived accuracy of information, reduced time to finalize discharge letter) $[41,48,49]$, and preventable adverse events [46]. Inconsistent effects were observed for various aspects of the use of care, namely, reduction of step-down unit length of stay (LOS), increase of transfers to higher care and increase of surgical procedures required [44, 45].

\section{Methodological quality}

The overall methodological quality of the studies was relatively low, with an average score of 4.5 points out of 
Fig. 1 Summary of evidence search and selection

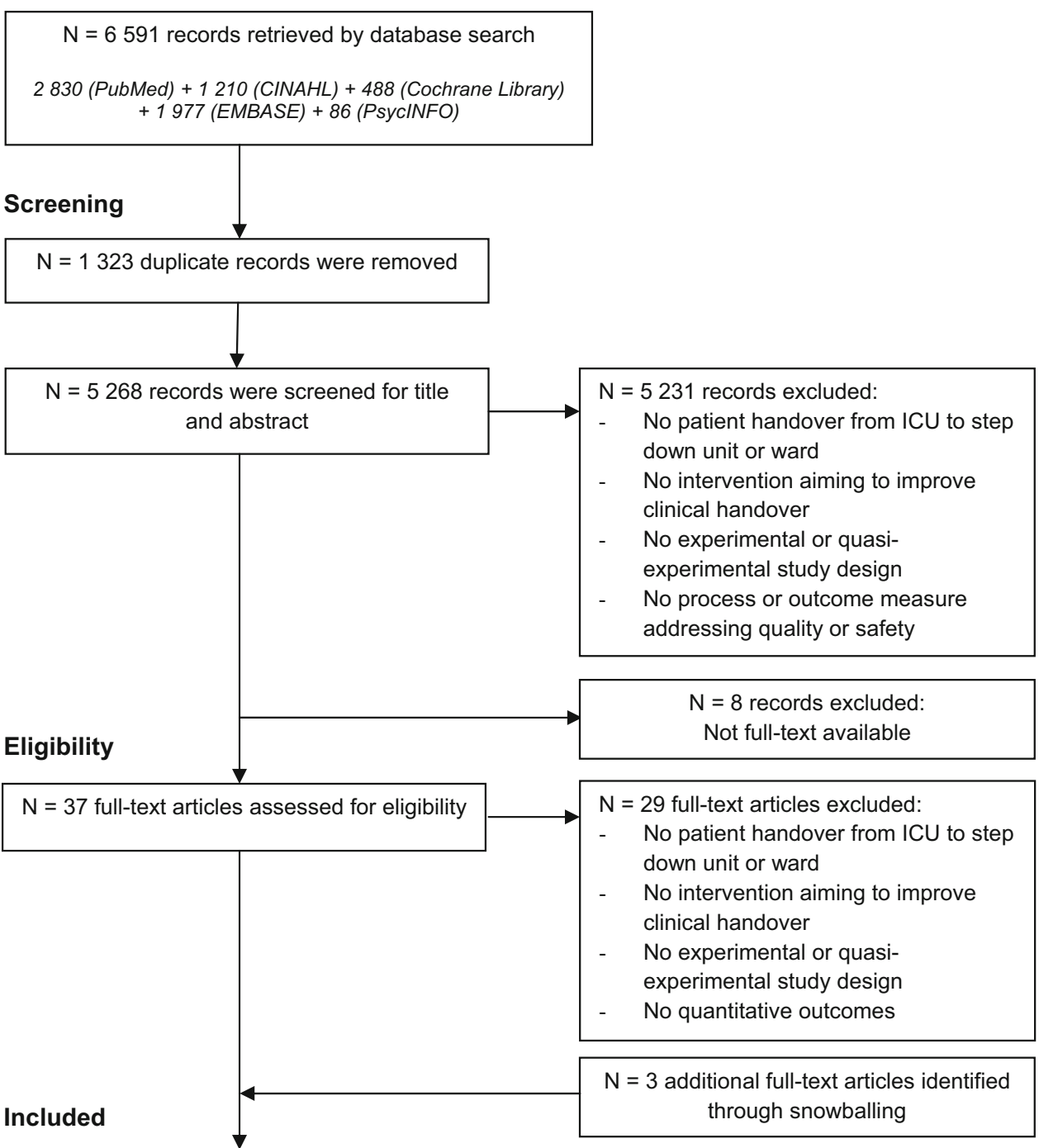

$\mathrm{N}=11$ full-text included in analysis
11 possible (ESM Table 2). In none of the studies was the allocation sequence randomly assigned and the allocation concealed. Six studies did not report similar baseline characteristics [40, 42, 43, 48-50], eight studies did not perform a sample size calculation [40-42, 44, 45, 48-50], and nine studies had no plan for handling missing data $[40,42-46,48-50]$.

\section{Classification and effects of interventions}

Table 3 provides an overview of the five types of interventions we identified in the 11 studies, namely, handover forms ( $n=3$ studies), a redesigned discharge process $(n=1)$, medication reconciliation $(n=1)$, liaison nurses $(n=4)$, and outreach services $(n=2)$.
In three of the 11 studies, handover forms as a tool for improving the information transferred between ICU and ward were evaluated [46, 48, 49]. All three studies found a statistically significant improvement in the reduction of adverse events [46] or in continuity of care [48, 49]. Williams and colleagues investigated the efficacy of a multidisciplinary form completed predominantly by nurses in combination with a discharge checklist completed by the medical staff. They found that the proportion of preventable adverse events was significantly reduced from 65 to $42 \%(p<0.001)$ [46]. Palma and colleagues implemented the use of a printed sign-out document and sign-out data entry form and reported that the staff perceived these new discharge tools to be significantly more accurate in terms of improving the transfer of information than those used previously $(p=0.0025)$ [48]. Medlock 


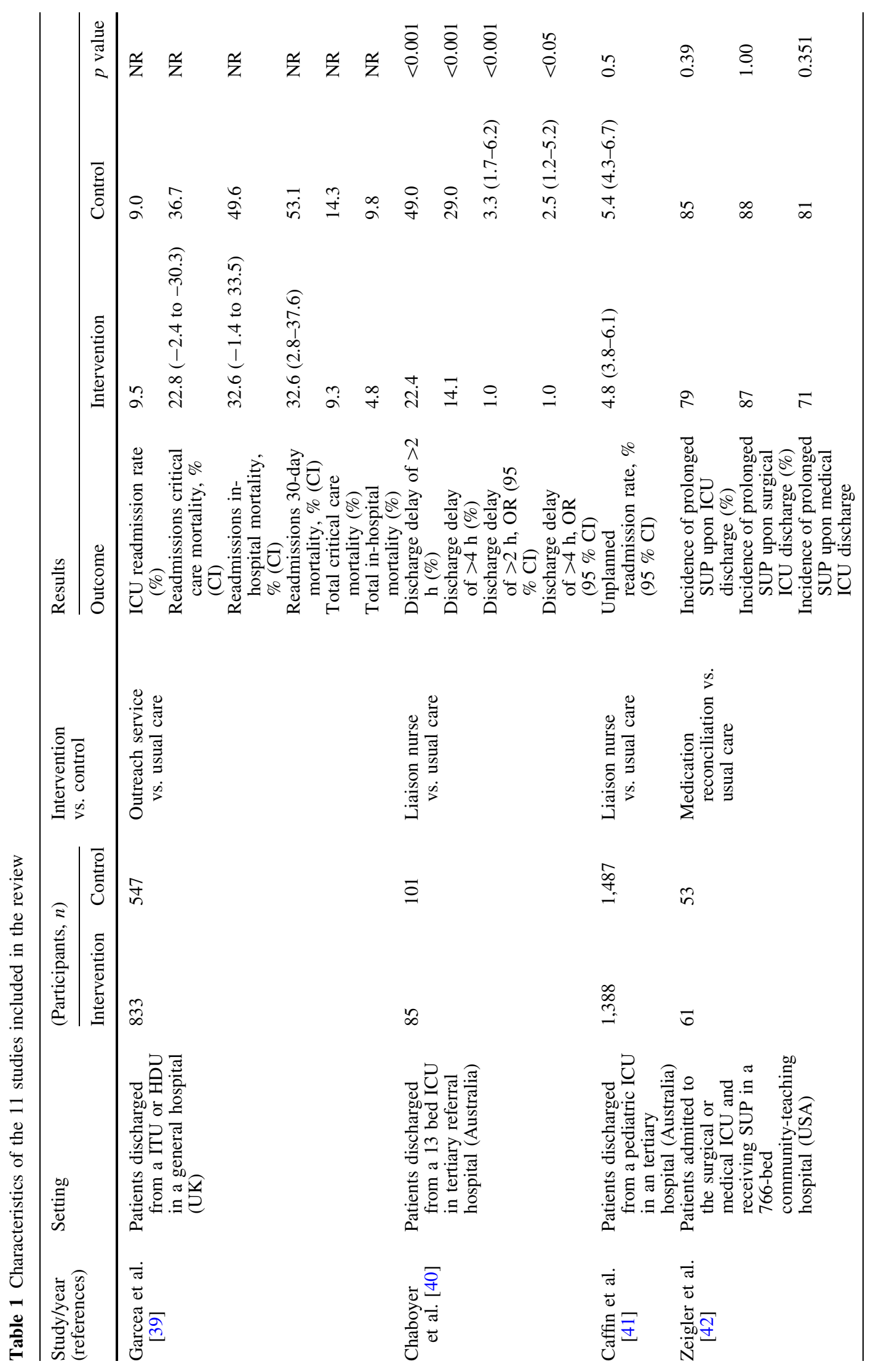




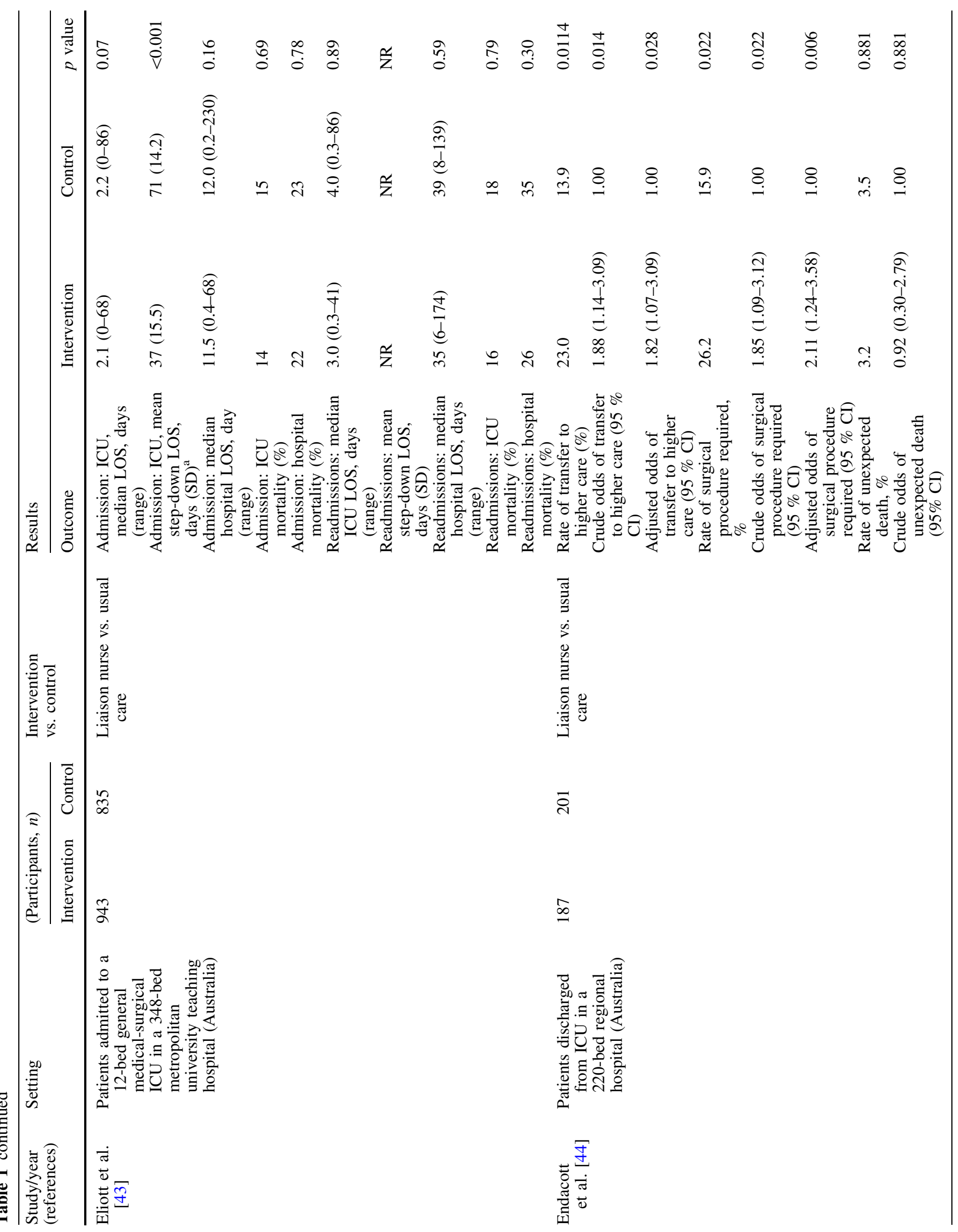




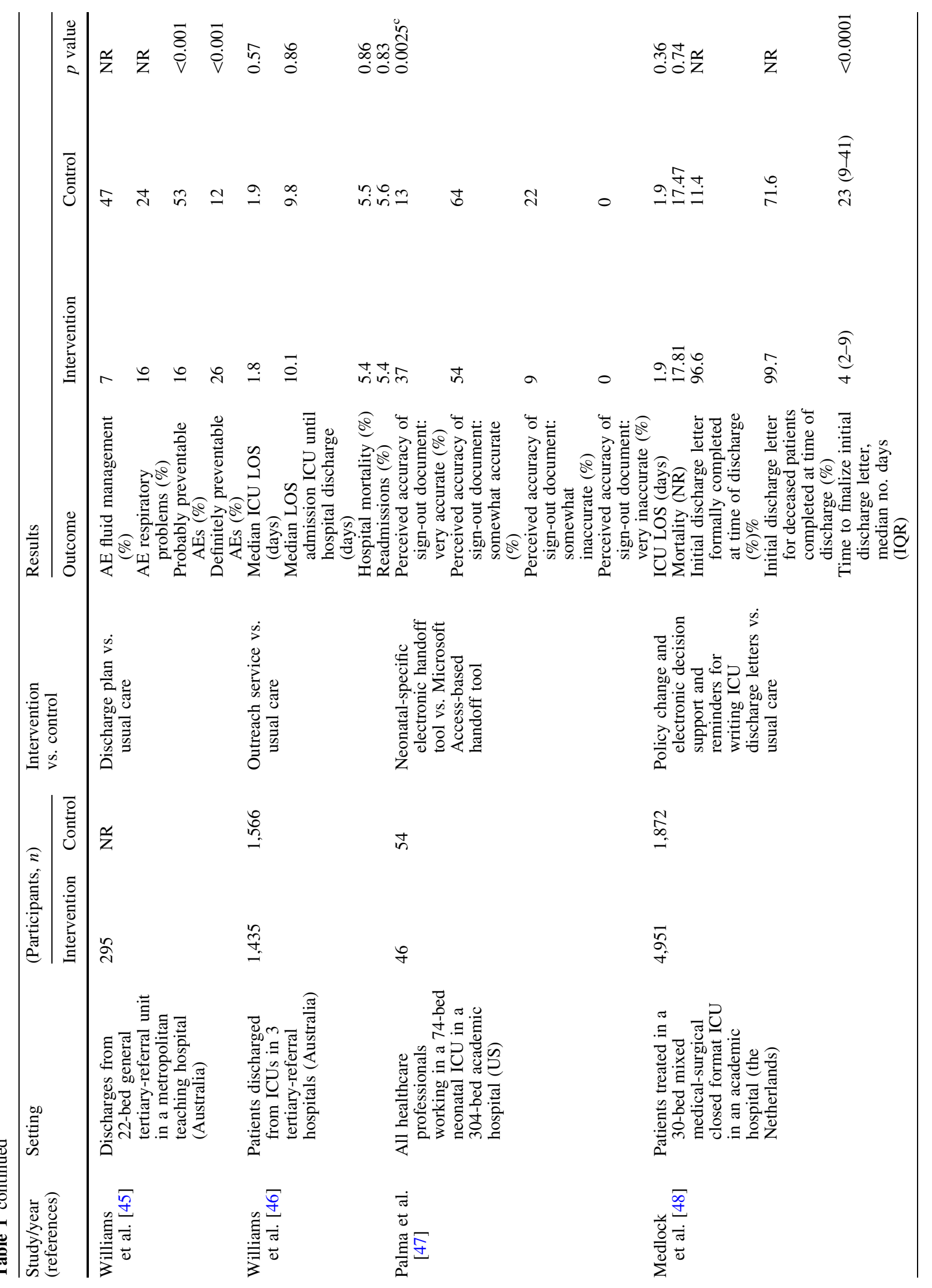




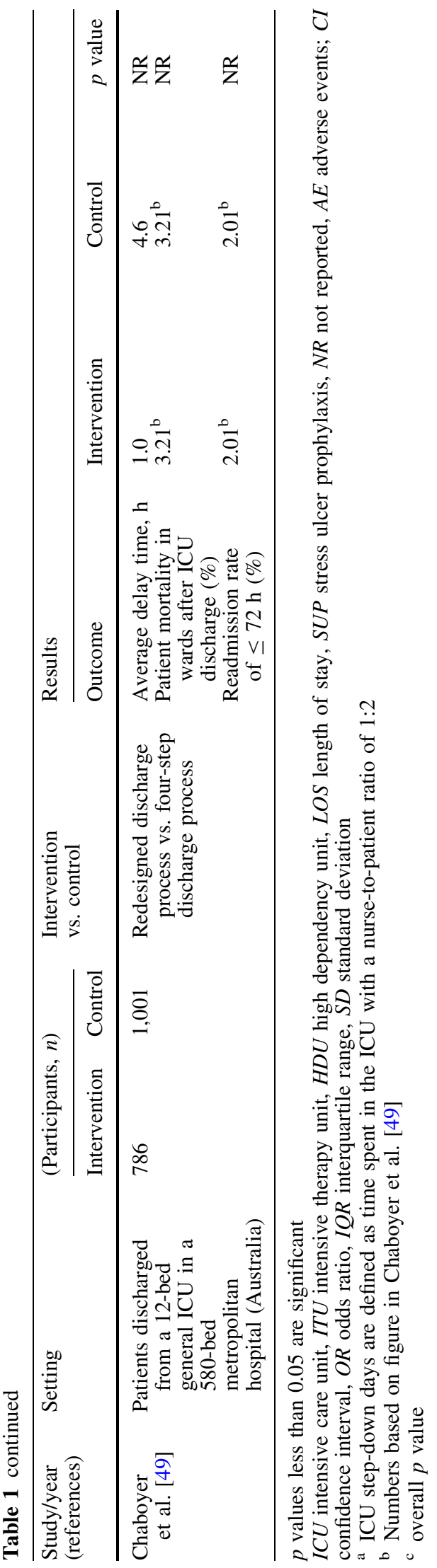

and colleagues investigated the implementation of an electronic discharge letter with a template to support content decisions. The median time to finalize the discharge letter was significantly reduced from 23 [interquartile range (IQR) 9-41) to 4 days (IQR 2-9; $p<0.0001$ ) [49].

Four studies examined the effects having an ICU liaison nurse in place to coordinate care and communication between ICU and general ward healthcare professionals [41, 42, 44, 45]. Three of these studies found that a liaison nurse had a statistically significant effect on use of care [44, 45] or continuity of care [41]. Chaboyer and colleagues evaluated the effects of liaison nurses who were involved in assessing patients for ICU discharge, coordinating transfer to other wards, and communicating with ward staff [41]. In their study, the liaison nurse assessed ward staff skill-mix and resources, prepared both the ICU and ward for transfer, assessed bed status, and provided clinical support and resources to ward nurses. The authors found that the proportion of patients with a discharge delay of $>2 \mathrm{~h}$ decreased significantly from 49 to $22 \%$ [odds ratio (OR) 3.3, $95 \%$ confidence interval (CI) 1.7-6.2, $p<0.001]$ and that the proportion with a discharge delay of $>4 \mathrm{~h}$ decreased significantly from 29 to $14 \%$ (OR $2.5,95 \%$ CI $1.2-5.2$, $p<0.05$ ) [41]. Elliott and colleagues implemented the use of liaison nurses who supported the management of discharged patients with complex care needs. The service involved communicating with ward staff and providing support and bedside education. These authors reported a significant reduction in mean step-down unit LOS from 71 to 37 days [44]. Endacott and colleagues investigated the role of a liaison nurse who visited patients at least daily for the first 3 days after ICU discharge [45]. In their study, the liaison nurse clinically assessed each patient, reviewed the charts, and provided support and informal education to ward staff. The proportion of patients discharged from the ICU who needed transfer to higher care was significantly increased from 14 to $23 \%$ (adjusted OR 1.82, $95 \%$ CI 1.07-3.09, $p=0.014$ ), and the proportion of patients requiring a surgical procedure significantly increased from 16 to $26 \%$ (adjusted OR 1.85, $95 \%$ CI 1.09-3.12, $p=0.022$ ) [45].

Two studies evaluated outreach services, in which activities were used which focused mainly on the followup of discharged ICU patients and supporting ward staff. Both studies found that the intervention did not have a statistically significant effect [40, 47].

Chaboyer and colleagues implemented a redesigned ICU discharge process, including a handover form to facilitate face-to-face or phone communication between ICU and ward healthcare professionals, a notification from the ward to their ICU counterparts of a specific time they were able to receive the patient, and a daily update to the ward staff summarizing all likely patient discharges 
Table 2 Outcome measures and statistical significance of effects reported in the 11 studies included in the review

\begin{tabular}{|c|c|c|c|c|c|}
\hline \multirow[t]{2}{*}{ Study/year (references) } & \multirow[t]{2}{*}{ Intervention } & \multicolumn{4}{|c|}{ Outcome types } \\
\hline & & $\begin{array}{l}\text { Use of } \\
\text { care }^{\mathrm{a}}\end{array}$ & $\begin{array}{l}\text { Continuity } \\
\text { of care }^{\mathrm{b}}\end{array}$ & Mortality $^{c}$ & $\begin{array}{l}\text { Adverse } \\
\text { events }\end{array}$ \\
\hline Garcea et al. [39] & Outreach service & $\boldsymbol{\nu}$ & & $\boldsymbol{V}$ & \\
\hline Chaboyer et al. [40] & Liaison nurse & & $\boldsymbol{\nu}^{\mathrm{e}}$ & & \\
\hline Caffin et al. [41] & Liaison nurse & $\checkmark$ & & & \\
\hline Zeigler et al. [42] & Medication reconciliation & $\checkmark$ & & & \\
\hline Eliott et al. [43] & Liaison nurse & $\boldsymbol{\nu}^{\mathrm{e}}$ & & $\boldsymbol{V}$ & \\
\hline Endacott et al. [44] & Liaison nurse & $\boldsymbol{\nu}^{\mathrm{e}}$ & & $\boldsymbol{\nu}$ & \\
\hline Williams et al. [45] & Discharge plan & & & & $\boldsymbol{\nu}^{\mathrm{e}}$ \\
\hline Williams et al. [46] & Outreach service & $\boldsymbol{\nu}$ & & $\boldsymbol{\nu}$ & \\
\hline Palma et al. [47] & Neonatal-specific electronic handoff tool & & $\boldsymbol{\nu}^{\mathrm{e}}$ & & \\
\hline Medlock et al. [48] & ICU discharge letter policy change and electronic decision support & $\boldsymbol{\nu}$ & $\boldsymbol{\nu}^{\mathrm{e}}$ & $\boldsymbol{V}$ & \\
\hline Chaboyer et al. [49] & Redesigned discharge process & $\boldsymbol{V}$ & $\boldsymbol{V}$ & $\checkmark$ & \\
\hline Total & & 9 & 4 & 7 & 1 \\
\hline
\end{tabular}

${ }^{\text {a }}$ Use of care as outcome includes (unplanned) readmissions; readmissions within $72 \mathrm{~h}$; ICU LOS; step-down LOS; general ward LOS; second ICU LOS; hospital LOS; LOS from admission to ICU to hospital discharge; transfer to higher level care; surgical procedure required; incidence of prolonged stress ulcer prophylaxis

b Continuity of care as outcome includes discharge delay ( $>2 \mathrm{~h} ;>4 \mathrm{~h}$ ); average delay time; initial discharge letter formally completed at time of discharge; initial discharge letter for deceased patient completed at time of discharge; time to finalize initial discharge letter; perceived accuracy of sign-out document (very accurate; somewhat accurate; somewhat inaccurate; very

('ICU discharge alert sheet') to better plan patient transfers and coordinate appropriate follow-up [50]. However, these authors reported that their changes to the ICU discharge process did have any statistically significant effects [50].

Zeigler and colleagues examined the use of medication reconciliation [43]. Upon ICU discharge, medication profiles were printed and reviewed by the primary physician and either discontinued or resumed. No statistically significant effects were found on the study outcome, namely, prolonged use of stress ulcer prophylaxis (SUP) [43].

\section{Implementation activities}

All of the 11 studies included in the review incorporated specific activities to facilitate the process of implementation of the intervention (Table 3). In terms of the implementation of handover forms, activities assessed to be effective were informal instructional sessions, the automatic filling of the handover form with data from the electronic medical record, development of software by the ICU staff, electronic reminders, a top-down directive, and involvement of healthcare professionals in the decisionmaking process $[46,48,49]$. Regarding the implementation of liaison nurses, activities assessed to be effective were a clear task description (based on the literature, inaccurate). Definitions adopted here are from Helles $\varnothing$ and colleagues [17] and are all outcomes that relate to the quality of information, communication, and coordination of care) [20]

c Mortality as outcome includes patient mortality in wards after ICU discharge; ICU mortality; critical care mortality; (in-) hospital mortality; 30-day mortality; unexpected death

d Incidence of adverse events (AE) as outcome includes AE fluid management; AE respiratory problems; probably preventable AEs; definitely preventable AEs-i.e., unintended occurrences in handover of care potentially causing harm to the patient [20]

e Outcome with statistically significant effect

formats of other hospitals, and experiences of patients, their families, and ICU and ward nurses), correct qualifications (experienced ICU or critical care nurse), provision of training to standardize the tasks carried out by liaison nurses, and encouraging ward staff to consult a liaison nurse if in doubt $[41,44,45]$.

\section{Discussion}

In this review we have reported the effects of interventions focusing on improving clinical handovers between ICU and ward healthcare professionals at the time of patient discharge from the ICU. After an extensive search process of six databases and subsequent selection of relevant reports, we ultimately only included 11 studies in our review, which indicates that very few studies on this specific topic have been performed. A statistically significant effect on quality of handover was observed in six of these studies $(55 \%)$. Effective interventions included: (1) implementation of liaison nurses to improve the communication and coordination of care between ICU and ward healthcare professionals and (2) handover forms to facilitate the timely handover of complete and accurate clinical information from ICU to ward healthcare professionals. Interventions were effective in improving the continuity of care and reducing preventable adverse 


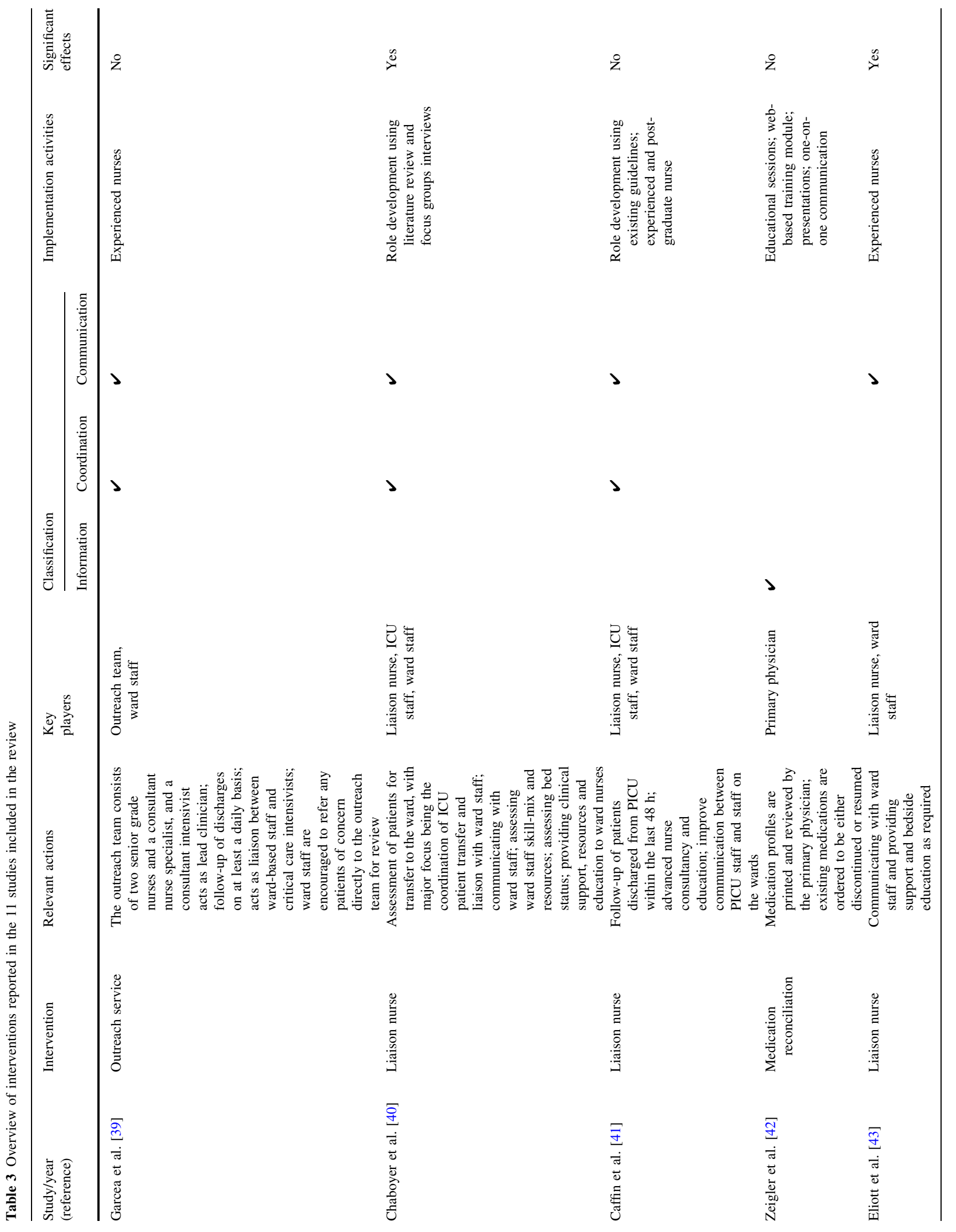




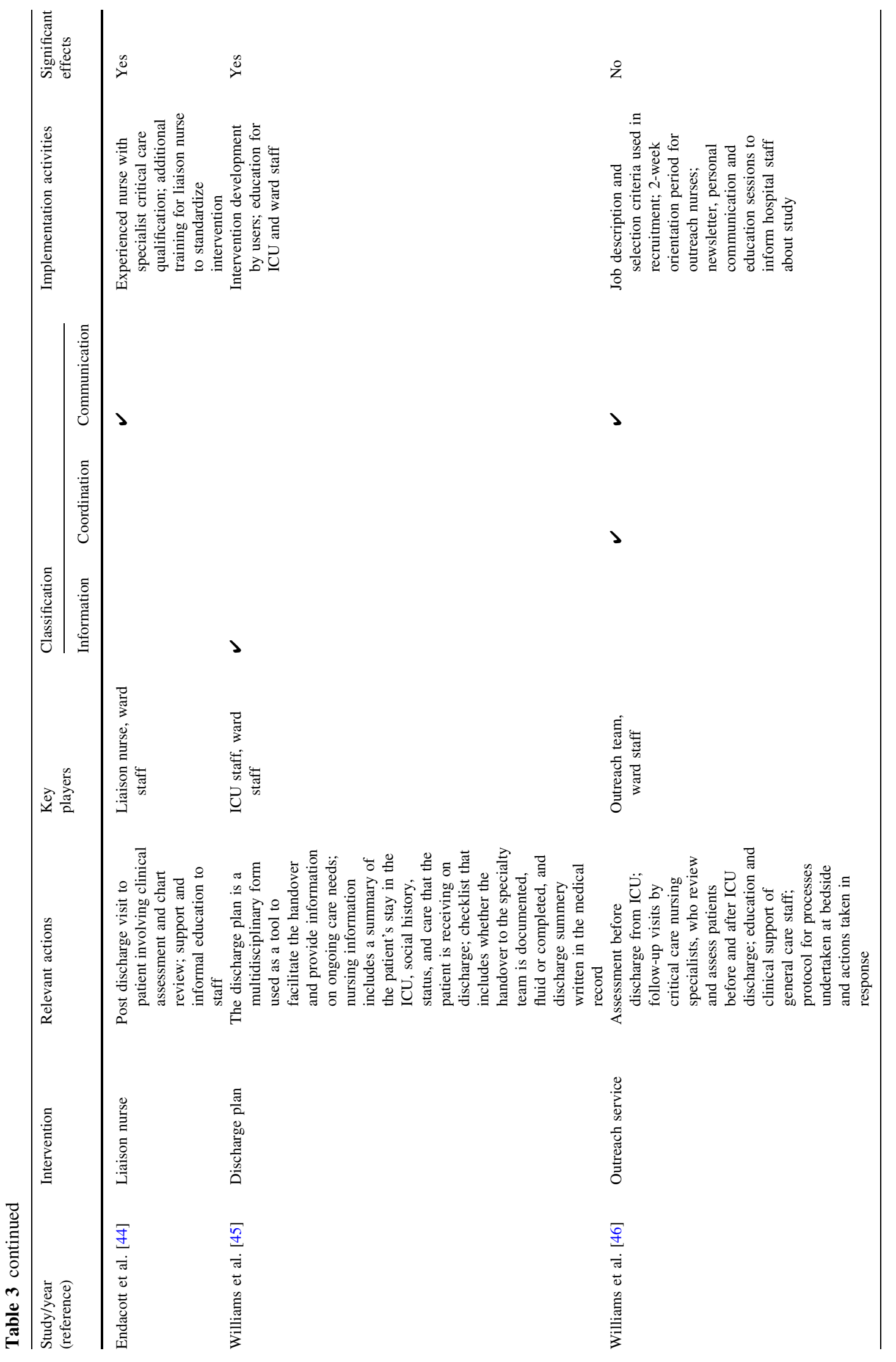




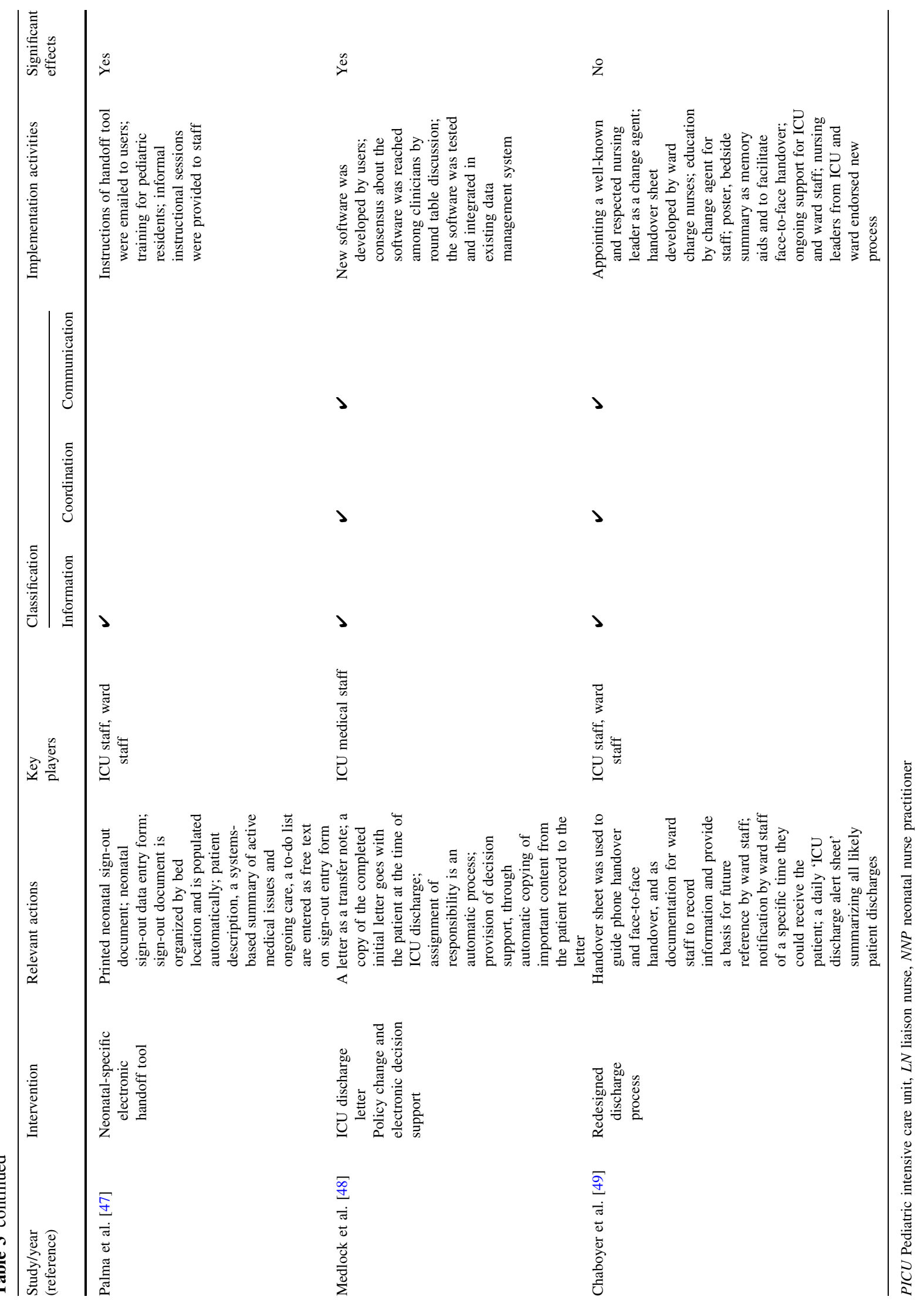


events. The effects found for the use of care were inconsistent; a decrease in step-down unit LOS was observed [44], as well as an increase in transfers to higher care and in the requirement for surgical procedures [45],

In accordance with our review, two recent studies report that liaison nurses can be a useful tool for bridging coordination gaps between healthcare settings $[20,30]$. In the studies included in this review, factors facilitating the implementation of a liaison nurse were a clear task description, 'casting' the right person based on experience, and encouragement of ward staff to consult a liaison nurse $[41,44,45]$. The literature also highlights a number of factors considered to be important for proper functioning of a liaison nurse: (1) that the liaison nurse be able to personalize his/her role as an ICU liaison nurse; (2) that the liaison nurse be able to gain the respect of ICU and general ward colleagues; (3) that ward staff view the implementation of a liaison nurse as a collaborative and supportive effort-and not as an intrusion in their ward [5].

Published studies also show that poor information transfer is a common patient safety issue in all types of handover settings [51-53]. Various reviews have reported the effectiveness of standardizing tools (e.g., standardized handoff tools, computerized handoff tools) to improve information transfer $[30,54,55]$ and, possibly, quality of care as well. In accordance with these studies, we found that the use of an ICU discharge form is an effective intervention by which to standardize information transfer and communication between ICU and ward healthcare professionals. It is interesting that the aims of the studies on information transfer focused on improving written communication [e.g., improving the situation background assessment recommendation (SBAR) checklist or collaboration between ICU and ward healthcare staff with team training], even though culture, team climate and verbal communication have been identified as important factors for inadequate patient handover [56].

Assessment of the 11 studies included in our systematic review reveals that a timely transfer of the patient together with accurate and complete information on the patient being transferred from the ICU to the ward are the specific aspects of handover which most readily show a change following interventions. Continuity of care in terms of reduced discharge delay and improved accuracy of discharge information was improved in three out of four studies investigating this outcome measure. However, whether this resulted in any beneficial clinical outcome beyond a better recording of data is unclear. Mortality rates were not improved in any of the seven studies evaluating mortality as an outcome measure. Evidence showing a reduction in ICU readmission or ICU LOS was limited; only one study found evidence for a reduction in step-down unit LOS [44].

The limitations of our review relate to the nature of the interventions and the study designs used. Similar to other reviews on patient handover, most interventions consisted of a complex set of activities. Most studies contain specific activities that have not been studied outside the set of activities used in the intervention. These aspects hinder an appropriate and direct evaluation of the interventions [20, 57]. Second, the poor methodological quality of most of the included studies makes it difficult to draw firm conclusions on the effectiveness of individual interventions. Single-institution evaluations with an observational design, i.e., non-controlled before-after design, dominated the studies we identified. In general, observational studies overestimate the effect. Third, the studies were characterized by significant heterogeneity for both interventions and outcome, making it impossible to perform a meta-analysis. Heterogeneity has been acknowledged to be a common limitation in the clinical handover literature $[9,20,54,57]$. Fourth, the classification of interventions into information, coordination, and communication categories was strictly based on the description of the intervention provided in the studies. Although interventions were independently classified by two researchers, the classification may be subject to bias due to minimal or unclear intervention descriptions. Fifth, we excluded nonpublished studies and non-full-text studies, which may have increased the risk of publication bias, i.e., the risk that this review overestimates or underestimates the true intervention effects. Moreover, we could not assess the risk of publication bias using a funnel plot due to the heterogeneity in outcome measures and the small number of studies found [39].

Despite handover being an important topic for the World Health Organization [58], and national government agencies, such as the Joint Commission, this systematic review highlights the absence of evidence on how to improve patient handovers between the ICU and general wards. Several reasons for the lack of effects have been described: use of an inappropriate intervention in relation to the underlying healthcare problem [43], measurement of inappropriate outcomes [50], and suboptimal research population, such as low mortality rate at baseline [47]. The lack of effects may also be influenced by limited actual exposure of healthcare professionals to the intervention and implementation activities [59]. These reasons reflect the difficulty of demonstrating the effectiveness of complex quality improvement interventions, as has been mentioned in several publications [60-62].

Our hope is that this systematic review will act as a stimulus to gather more evidence on the interventions described in the 11 studies included in the review, as well as interventions evaluated in other settings, such as a shared electronic information exchange system to improve handover between hospital and primary healthcare providers [63]. The implementation of interventions for which insufficient evidence is available carries the burden of potentially wasting valuable resources, which may increase the reluctance of clinicians to implement 
other quality improvement initiatives [64]. We recommend that researchers and clinicians considering to conduct an evaluation of an improved handover process use robust designs to strengthen the quality of evidence on this topic. Randomized controlled trials are often impossible to conduct due to difficulties in blinding and concealment of allocation. Cluster randomized controlled trials pose difficulties in terms of sample size and obtaining a uniform control group. Other rigorous study designs, such as an interrupted time-series or a controlled before-and-after study, are good alternatives and are more feasible in practice [65]; however, they are associated with a greater risk of bias. Objective outcome or performance measures, such as readmission rate or mortality rate, are the ideal parameters for measuring effectiveness, but due to low incidence, it is hard to reach statistical significance. Process measures can be used to gain more insight in the processes leading to improvement in the outcome measures [61].
In conclusion, liaison nurses and handover forms are promising interventions to improve the quality of clinical handover between ICU and ward healthcare professionals at patient discharge. Due to the limited number of studies identified in this review and the weak methodological quality of the included studies, more robust evidence is needed.

\section{Conflicts of interest None.}

Ethical standard Ethical approval was not required under Dutch National Law.

Open Access This article is distributed under the terms of the Creative Commons Attribution Noncommercial License which permits any noncommercial use, distribution, and reproduction in any medium, provided the original author(s) and the source are credited.

\section{References}

1. Halpern NA, Pastores SM (2010) Critical care medicine in the United States 2000-2005: an analysis of bed numbers, occupancy rates, payer mix, and costs. Crit Care Med 38:65-71

2. Tan SS, Bakker J, Hoogendoorn ME, Kapila A, Martin J, Pezzi A, Pittoni G, Spronk PE, Welte R, Hakkaart-van Roijen L (2012) Direct cost analysis of intensive care unit stay in four European countries: applying a standardized costing methodology. Value Health 15:81-86

3. Cook R, Rasmussen J (2005) "Going solid": a model of system dynamics and consequences for patient safety. Qual Saf Health Care 14:130-134

4. Lin F, Chaboyer W, Wallis M (2009) A literature review of organisational, individual and teamwork factors contributing to the ICU discharge process. Aust Crit Care 22:29-43

5. Chaboyer W, James H, Kendall M (2005) Transitional care after the intensive care unit: current trends and future directions. Crit Care Nurse 25:16-29

6. Häggstrom M, Asplund K, Kristiansen L (2009) Struggle with a gap between intensive care units and general wards. Int J Qual Stud Health Well-being 4:181-192

7. Häggstrom M, Asplund K, Kristiansen L (2012) How can nurses facilitate patient's transitions from intensive care?: a grounded theory of nursing. Intensive Crit Care Nurs 28:224-233
8. Greenberg CC, Regenbogen SE, Studdert DM, Lipsitz SR, Rogers SO, Zinner MJ, Gawande AA (2007) Patterns of communication breakdowns resulting in injury to surgical patients. J Am Coll Surg 204:533-540

9. Niven DJ, Bastos JF, Stelfox HT (2013) Critical care transition programs and the risk of readmission or death after discharge from an ICU: a systematic review and meta-analysis. Crit Care Med 42:179-187

10. Stelfox HT, Perrier L, Straus SE, Ghal WA, Zygun D, Boiteau P, Zuege DJ (2013) Identifying intensive care unit discharge planning tools: protocol for a scoping review. BMJ Open 3:e002653

11. Daly K, Beale R, Chang RW (2001) Reduction in mortality after inappropriate early discharge from intensive care unit: logistic regression triage model. BMJ 322:1274-1276

12. Society of Critical Care Medicine (1999) Guidelines for intensive care unit admission, discharge, and triage. Crit Care Med 27:633-638

13. Nederlandse Vereniging voor Intensive Care (2011) Criteria voor opname en ontslag van Intensive Care afdelingen in Nederland. Available at: http://nvic.nl/sites/default/files/ Richtlijnen\%20aanmaken/ Herziene\% 20richtlijn\%20 Opname \%20en\%20ontslag\%20 criteria\%20januari\%202011.pdf. Accessed 18 Sept 2014
14. Intensive Care Society (2013) Core standards for intensive care units. Available at: http://www.ficm. ac.uk/sites/default/files/Core\% 20Standards $\% 20$ for $\% 20$ ICUs $\% 20 \mathrm{Ed}$. $1 \% 20 \% 282013 \% 29$.pdf. Accessed 18 Sept 2014

15. Heidegger $\mathrm{CP}$, Treggiari MM, Romand JA, Swiss ICU Network (2005) A nationwide survey of intensive care unit discharge practices. Intensive Care Med 31:1676-1682

16. Nishi GK, Suh RH, Wilson MT, Cunneen SA, Margulies DR, Shabot MM (2003) Analysis of causes and prevention of early readmission to surgical intensive care. Am Surg 69:913-917

17. Helles $\varnothing$ R, Lorensen M, Sorensen L (2004) Challenging the information gap-the patients transfer from hospital to home health care. Int $\mathbf{J}$ Med Inform 73:569-580

18. Perren A, Conte P, De Bitonti N, Limoni C, Merlani P (2008) From the ICU to the ward: cross-checking of the physician's transfer report by intensive care nurses. Intensive Care Med 34:2054-2061

19. Li P, Stelfox HT, Ghali WA (2011) A prospective observational study of physician handoff for intensive-careunit-to-ward patient transfers. Am J Med 124:860-867 
20. Hesselink G, Schoonhoven L, Barach P, Spijker A, Gademan P, Kalkman C, Liefers J, Vernooij-Dassen M, Wollersheim H (2012) Improving patient handovers from hospital to primary care: a systematic review. Ann Intern Med 157:417-428

21. Russell S (1999) Reducing readmissions to the intensive care unit. Heart Lung 28:365-372

22. Whittaker J, Ball C (2000) Discharge from intensive care: a view from the ward. Intensive Crit Care Nurs 16:135-143

23. Kramer AA, Higgins TL, Zimmerman JE (2013) The association between ICU readmission rate and patient outcomes. Crit Care Med 41:24-33

24. Rosenberg AL, Hofer TP, Hayward RA, Strachan C, Watts CM (2001) Who bounces back? Physiologic and other predictors of intensive care unit readmission. Crit Care Med 29:511-518

25. Angus DC (2007) Caring for the critically ill patient: challenges and opportunities. JAMA 298:456-458

26. Dawson S, King L, Grantham H (2013) Review article: improving the hospital clinical handover between paramedics and emergency department staff in the deteriorating patient. Emerg Med Australas 25:393-405

27. Jensen SM, Lippert A, Ostergaard D (2013) Handover of patients: a topical review of ambulance crew to emergency department handover. Acta Anaesthesiol Scand 57:964-970

28. DeRienzo CM, Frush K, Barfield ME, Gopwani PR, Griffith BC, Jiang X, Mehta AI, Papavassiliou P, Rialon KL, Stephany AM, Zhang T, Andolsek KM (2012) Handoffs in the era of duty hours reform: a focused review and strategy to address changes in the Accreditation

Council for Graduate Medical Education Common Program Requirements. Acad Med 87:403-410

29. Flemming D, Hubner U (2013) How to improve change of shift handovers and collaborative grounding and what role does the electronic patient record system play? Results of a systematic literature review. Int J Med Inform 82:580-592

30. Kripalani S, LeFevre F, Phillips CO, Williams MV, Basaviah P, Baker DW (2007) Deficits in communication and information transfer between hospitalbased and primary care physicians: implications for patient safety and continuity of care. JAMA 297:831-841

31. Møller TP, Madsen MD, Fuhrmann L, Ostergaard D (2013) Postoperative handover: characteristics and considerations on improvement: a systematic review. Eur J Anaesthesiol 30:229-242
32. Lai JI, Lin HY, Lai YC, Lin PC, Chang SC, Tang GJ (2012) Readmission to the intensive care unit: a population-based approach. J Formos Med Assoc 111:504-509

33. Araujo TG, Rieder Mde M, Kutchak FM, Franco Filho JW (2013) Readmissions and deaths following ICU discharge: a challenge for intensive care. Rev Bras Ter Intensiva 25:32-38

34. Benetis R, Sirvinskas E, Kumpaitiene B, Kinduris S (2013) A case-control study of readmission to the intensive care unit after cardiac surgery. Med Sci Monit 19:148-152

35. van Sluisveld N, Zegers M, Westert G, van der Hoeven JG, Wollersheim $\mathrm{H}$ (2013) A strategy to enhance the safety and efficiency of handovers of ICU patients: study protocol of the pICUp study. Implement Sci 8:67

36. Liberati A, Altman DG, Tetzlaff J, Mulrow C, Gotzsche PC, Ioannidis JP, Clarke M, Devereaux PJ, Kleijnen J, Moher D (2009) The PRISMA statement for reporting systematic reviews and meta-analyses of studies that evaluate health care interventions: explanation and elaboration. Ann Intern Med 151:W65-W94

37. Esmonde L, McDonnell A, Ball C, Waskett C, Morgan R, Rashidian A, Bray K, Adam S, Harvey S (2006) Investigating the effectiveness of critical care outreach services: a systematic review. Intensive Care Med 32:1713-1721

38. Grimshaw J, McAuley LM, Bero LA, Grilli R, Oxman AD, Ramsay C, Vale L, Zwarenstein M (2003) Systematic reviews of the effectiveness of quality improvement strategies and programmes. Qual Saf Health Care 12:298-303

39. Higgins J, Green S (2006) Cochrane handbook for systematic reviews of interventions. J Wiley, Chichester

40. Garcea G, Thomasset S, McClelland L, Leslie A, Berry DP (2004) Impact of a critical care outreach team on critical care readmissions and mortality. Acta Anaesthesiol Scand 48:1096-1100

41. Chaboyer W, Thalib L, Foster M, Elliott D, Endacott R, Richards B (2006) The impact of an ICU liaison nurse on discharge delay in patients after prolonged ICU stay. Anaesth Intensive Care 34:55-60

42. Caffin CL, Linton S, Pellegrini J (2007) Introduction of a liaison nurse role in a tertiary paediatric ICU. Intensive Crit Care Nurs 23:226-233

43. Zeigler AJ, McAllen KJ, Slot MG, Barletta JF (2008) Medication reconciliation effect on prolonged inpatient stress ulcer prophylaxis. Ann Pharmacother 42:940-946
44. Eliott SJ, Ernest D, Doric AG, Page KN, Worrall-Carter LJ, Thalib L, Chaboyer W (2008) The impact of an ICU liaison nurse service on patient outcomes. Crit Care Resusc 10:296-300

45. Endacott R, Chaboyer W, Edington J, Thalib L (2010) Impact of an ICU Liaison Nurse Service on major adverse events in patients recently discharged from ICU. Resusc 81:198-201

46. Williams TA, Leslie GD, Elliott N, Brearley L, Dobb GJ (2010) Introduction of discharge plan to reduce adverse events within 72 hours of discharge from the ICU. J Nurs Care Qual 25:73-79

47. Williams TA, Leslie G, Finn J, Brearley L, Asthifa M, Hay B, Laurie K, Leen T, O'Brien K, Stuart M, Watt M (2010) Clinical effectiveness of a critical care nursing outreach service in facilitating discharge from the intensive care unit. Am J Crit Care 19:e63-e72

48. Palma JP, Sharek PJ, Longhurst CA (2011) Impact of electronic medical record integration of a handoff tool on sign-out in a newborn intensive care unit. J Perinatol 31:311-317

49. Medlock S, Eslami S, Askari M, van Lieshout EJ, Dongelmans DA, AbuHanna A (2011) Improved communication in post-ICU care by improving writing of ICU discharge letters: a longitudinal before-after study. BMJ Qual Saf 20:967-973

50. Chaboyer W, Lin F, Foster M, Retallick L, Panuwatwanich K, Richards B (2012) Redesigning the ICU nursing discharge process: a quality improvement study. Worldviews Evid Based Nurs 9:40-48

51. Horwitz LI, Moin T, Krumholz HM, Wang L, Bradley EH (2008) Consequences of inadequate sign-out for patient care. Arch Intern Med 168:1755-1760

52. Horwitz LI, Meredith T, Schuur JD, Shah NR, Kulkarni RG, Jenq GY (2009) Dropping the baton: a qualitative analysis of failures during the transition from emergency department to inpatient care. Ann Emerg Med 53:701-710

53. Horwitz LI, Moin T, Krumholz HM, Wang L, Bradley EH (2009) What are covering doctors told about their patients? Analysis of sign-out among internal medicine house staff. Qual Saf Health Care 18:248-255

54. Foster S, Manser T (2012) The effects of patient handoff characteristics on subsequent care: a systematic review and areas for future research. Acad Med 87:1105-1124

55. Li P, Ali S, Tang C, Ghali WA, Stelfox HT (2013) Review of computerized physician handoff tools for improving the quality of patient care. J Hosp Med 8:456-463 
56. Hesselink G, Vernooij-Dassen M, Pijnenborg L, Barach P, Gademan P, Dudzik-Urbaniak E, Flink M, Orrego C, Toccafondi G, Johnson JK, Schoonhoven L, Wollersheim H, European HANDOVER Research Collaborative (2013) Organizational culture: an important context for addressing and improving hospital to community patient discharge. Med Care 51:90-98

57. Hansen LO, Young RS, Hinami K, Leung A, Williams MV (2011) Interventions to reduce 30-day rehospitalization: a systematic review. Ann Intern Med 155:520-528

58. World Health Organization (WHO) Collaborating Centre for Patient Safety Sollutions (2007) Comunication during patient hand-overs. Available at: http://www.who.int/patientsafety/ solutions/patientsafety/PS-

Solution3.pdf. Accessed 18 Sept 2014
59. Hulscher ME, Laurant MG, Grol RP (2003) Process evaluation on quality improvement interventions. Qual Saf Health Care 12:40-46

60. Clancy CM, Berwick DM (2011) The science of safety improvement: learning while doing. Ann Intern Med 154:699-701

61. Lilford RJ, Chilton PJ, Hemming K, Girling AJ, Taylor CA, Barach P (2010) Evaluating policy and service interventions: framework to guide selection and interpretation of study end points. BMJ 341:c4413

62. Shekelle PG, Pronovost PJ, Wachter RM, Taylor SL, Dy SM, Foy R, Hempel $\mathrm{S}$, McDonald KM, Ovretveit J, Rubenstein LV, Adams AS, Angood PB, Bates DW, Bickman L, Carayon P, Donaldson L, Duan N, Farley DO, Greenhalgh T, Haughom J, Lake ET, Lilford R, Lohr KN, Meyer GS, Miller MR, Neuhauser DV, Ryan G, Saint S, Shojania KG, Shortell SM, Stevens DP Walshe K (2011) Advancing the science of patient safety. Ann Intern Med 154:693-696
63. Hesselink G, Zegers M, VernooijDassen M, Barach P, Kalkman C, Flink M, Ohlen G, Olsson M, Bergenbrant S, Orrego C, Sunol R, Toccafondi G, Venneri F, Dudzik-Urbaniak E, Kutryba B, Schoonhoven L, Wollersheim H, European HRC (2014) Improving patient discharge and reducing hospital readmissions by using intervention mapping. BMC Health Serv Res 14:389

64. Smeulers M, Lucas C, Vermeulen H (2014) Effectiveness of different nursing handover styles for ensuring continuity of information in hospitalised patients. Cochrane Database Syst Rev 6:CD009979

65. Shojania KG (2013) Conventional evaluations of improvement interventions: more trials or just more tribulations? BMJ Qual Saf 22:881-884 